\title{
Prevalence of diabetes and impaired fasting glucose in the Chinese adult population: International Collaborative Study of Cardiovascular Disease in Asia (InterASIA)
}

\author{
D. Gu${ }^{1}$, K. Reynolds 2 , X. Duan ${ }^{1}$, X. Xin ${ }^{2}$, J. Chen ${ }^{3}$, X. Wu ${ }^{1}$, J. Mo ${ }^{4}$, P. K. Whelton ${ }^{2,3}$, J. He ${ }^{2,3}$ for the InterASIA \\ Collaborative Group \\ ${ }^{1}$ Cardiovascular Institute and Fuwai Hospital of Chinese Academy of Medical Sciences, Peking Union Medical College, Beijing, \\ China \\ 2 Department of Epidemiology, Tulane University School of Public Health and Tropical Medicine, New Orleans, USA \\ 3 Tulane University School of Medicine, New Orleans, Louisiana, USA \\ ${ }^{4}$ Pfizer Inc, New York, NY, USA
}

\section{Abstract}

Aims/hypothesis. To estimate the prevalence of diagnosed and undiagnosed diabetes and impaired fasting glucose in the general adult population of China.

Methods. The International Collaborative Study of Cardiovascular Disease in ASIA, conducted from 2000 to 2001, included a nationally representative sample of 15540 adults, aged 35 to 74 years. An overnight fasting blood specimen was collected to measure serum glucose and information on history of diabetes and use of hypoglycaemic medications was obtained by a standard questionnaire. Undiagnosed diabetes (fasting glucose $\geq 7.0 \mathrm{mmol} / \mathrm{l}$ ) and impaired fasting glucose $(6.1-6.9 \mathrm{mmol} / \mathrm{l})$ were defined using the American Diabetes Association criteria.

Results. Prevalence of self-reported diagnosed diabetes, undiagnosed diabetes, and impaired fasting glucose in Chinese adults were $1.3 \%, 4.2 \%$, and $7.3 \%$, respectively. Overall, $5.2 \%$ or 12.7 million men and $5.8 \%$ or 13.3 million women in China aged 35 to 74 years had diabetes (self-reported diagnosis plus undiagnosed diabetes). The age-standardized prevalence of diabetes was higher in residents of northern compared to southern China ( $7.4 \%$ vs $5.4 \%, p<0.001)$ and in those living in urban compared to rural areas $(7.8 \%$ vs $5.1 \%, p<0.001)$.

Conclusions/interpretation. Our results show that the prevalence of diabetes in the adult population in China is much higher than previously reported. Three out of every four diabetes patients are undiagnosed, indicative of a lack of population-based screening programmmes and a relatively rapid and recent increase in incidence of diabetes. These results indicate that diabetes has become a major public health problem in China and underscore the need for national strategies aimed at prevention and treatment of diabetes. [Diabetologia (2003) 46:1190-1198]

Keywords Diabetes Mellitus, glucose, prevalence, cross-sectional studies, China.
Received: 23 December 2002 / Revised: 28 May 2003

Published online: 15 July 2003

(C) Springer-Verlag 2003

Corresponding author: J. He, Department of Epidemiology, Tulane University School of Public Health and Tropical Medicine, 1430 Tulane Avenue SL18, New Orleans, Louisiana 70112, USA

E-mail: jhe@tulane.edu

Abbreviations: InterASIA, International Collaborative Study of Cardiovascular Disease in ASIA; ADA, American Diabetes Association; WHO, World Health Organization; DECODE, Diabetes Epidemiology: Collaborative Analysis of Diagnostic Criteria in Europe.
Diabetes is a well-established modifiable risk factor for cardiovascular and renal diseases in Western countries $[1,2]$. The importance of diabetes is likely to increase because the prevalence of diabetes, particularly Type 2 diabetes, has risen steadily during recent decades and is projected to continue to increase. The prevalence of diabetes in a recent survey in the U.S. was $7.8 \%$ in adults aged 20 years and older, with a further $6.9 \%$ recording an impaired fasting glucose concentration [3]. The increasing prevalence of diabetes in the U.S. population contrasts with the observed decline in the prevalence of hypertension and hypercholesterolaemia in the same populations $[4,5]$. The rising prevalence of obesity, related to caloric intake in excess of energy expenditure, is a likely cause for 
the increase in the prevalence of diabetes [6]. An estimated 300 million adults worldwide will have diabetes by the year 2025 [7].

Diabetes has become an important public health challenge in economically developing countries, especially in Asian countries [7]. Several large cross-sectional surveys have yielded prevalence estimates for diabetes in mainland China $[8,9,10]$. The prevalence of Type 2 diabetes and impaired glucose tolerance was reported to be about $1 \%$ each among a sample of 110660 men and women aged 25 to 74 years living in an urban community in 1986 [8, 9]. The 1994 China National Diabetes Survey examination of 224251 men and women aged 25 to 64 years yielded prevalence estimates for diabetes and impaired glucose tolerance of $2.5 \%$ and $3.2 \%$, respectively [10]. Neither of these surveys, however, was based on experience from a representative national sample [8, 9, 10]. The 1986 survey was conducted in only one industrial city in northern China while in the 1994 survey, the selection of study participants was dependent upon the willingness of local physicians to collaborate in the study.

The International Collaborative Study of Cardiovascular Disease in Asia (InterASIA) was designed to provide current and reliable data on the prevalence of selected cardiovascular disease risk factors, including diabetes and impaired fasting glucose, in the adult population in China.

\section{Subjects and methods}

Study population. A four-stage stratified sampling method was used to select a nationally representative sample of the general population aged 35 to 74 years in China. The sampling process was stratified by geographic characteristics (rural vs urban areas and northern vs southern China). In stage one, 31 provinces (and municipalities) were stratified into northern and southern China, as divided by the Yangtzi River. Five provinces or municipalities from northern China and five from southern China were selected to be representative of the geographic and economic developing status in their regions. One rural county and one city region from each selected province or municipality were randomly selected and one township or one street district (about 1000-2000 households) was randomly selected from each of the counties and city regions, respectively. In the final stage, individuals aged 35 to 74 years were randomly selected from the selected township or street district. Only one participant was selected from each household, without replacement.

A total of 19012 people were selected and invited to participate in the survey. A total of 15838 people (7684 men and 8154 women) completed the study. The overall response rate was $83.3 \%$ (82.1\% in men and $84.5 \%$ in women; $82.2 \%$ in urban and $84.4 \%$ in rural areas; and $82.9 \%$ in northern China and $83.8 \%$ in southern China). The present analysis was restricted to the 15540 adults who were aged 35 to 74 years at the time of the survey. Fasting serum glucose values were obtained from 15236 adults.

Data collection. Data collection was conducted in examination centres at local health stations or community clinics in the par- ticipants' residential area. In a few instances, participants were unable to attend the examination centre and the interview and examination were conducted in their home. During the clinic or home visits, trained research staff administered a standard questionnaire. Information on demographic characteristics, including age, sex, education, and occupation was collected. The interview included questions related to the diagnosis and treatment of diabetes. Women were asked whether diabetes had been diagnosed during pregnancy and/or whether it had been diagnosed when they were not pregnant.

An overnight-fasting venous blood specimen for measurement of serum glucose was collected using a vacuum tube containing sodium fluoride. The fasting time was verified prior to collecting the blood specimen. Participants who had not fasted for at least $10 \mathrm{~h}$ did not have their blood drawn. Blood specimens were processed at the examination centre and shipped by air to a central clinical laboratory in Beijing, where the specimens were stored at $-70^{\circ} \mathrm{C}$ until laboratory assays could be carried out. Serum glucose was measured using a modified hexokinase enzymatic method (HITACHI automatic clinical analyzer, Model 7060, Japan).

Participants without a prior diagnosis of diabetes were categorized according to the American Diabetes Association (ADA) diagnostic criteria as follows: undiagnosed diabetes (fasting glucose $\geq 7.0 \mathrm{mmol} / \mathrm{l}$ ) and impaired fasting glucose (6.1-6.9 mmol/1). Diabetes was defined as a self-reported history of diabetes plus undiagnosed diabetes [11]. All study participants were asked to bring in samples of their prescription medications during the clinic visit. A self-reported history of diabetes was confirmed by the use of insulin or oral hypoglycaemic agents. In addition, hospital chart reviews were conducted for all hospitalized cases of diabetes.

All study investigators and staff members successfully completed a training programmme that oriented them both to the aims of the study and to the specific tools and methodologies employed. At the training sessions, interviewers were given detailed instructions on administration of the study questionnaire.

The Institutional Review Board at the Tulane University Health Sciences Centre approved the InterASIA study. In addition, ethics committees and other relevant regulatory bodies in China approved the study. Informed consent was obtained from each participant prior to data collection.

Statistical methods. The InterASIA study was designed to provide precise estimates of prevalence and mean glucose concentrations for both men and women in rural and urban areas, and in northern and southern China among the following four age groups: 35 to 44,45 to 54,55 to 64 and 65 to 74 years old. The size of the InterASIA sample was selected to meet generally recommended requirements for precision in a complex survey [12].

All calculations were weighted to represent the total Chinese adult population aged 35 to 74 years. The weighting was calculated based on data from the year 2000 China Population Census and the InterASIA sampling scheme. Separate weightings were used for the samples who were interviewed (participants with a self-reported history of diabetes) and for the sample whose diagnosis was based on measurement of serum glucose (participants with undiagnosed diabetes or impaired fasting glucose). Standard errors were calculated by a technique appropriate to the complex survey design. Age-standardized estimates of prevalence were calculated by the direct method using the year 2000 China adult population aged 35 to 74 as the standard population. Estimated numbers of diabetes and impaired fasting glucose were calculated using age-specific prevalence and data from the year 2000 China Population 
Table 1. Study participant characteristics by geographic regions

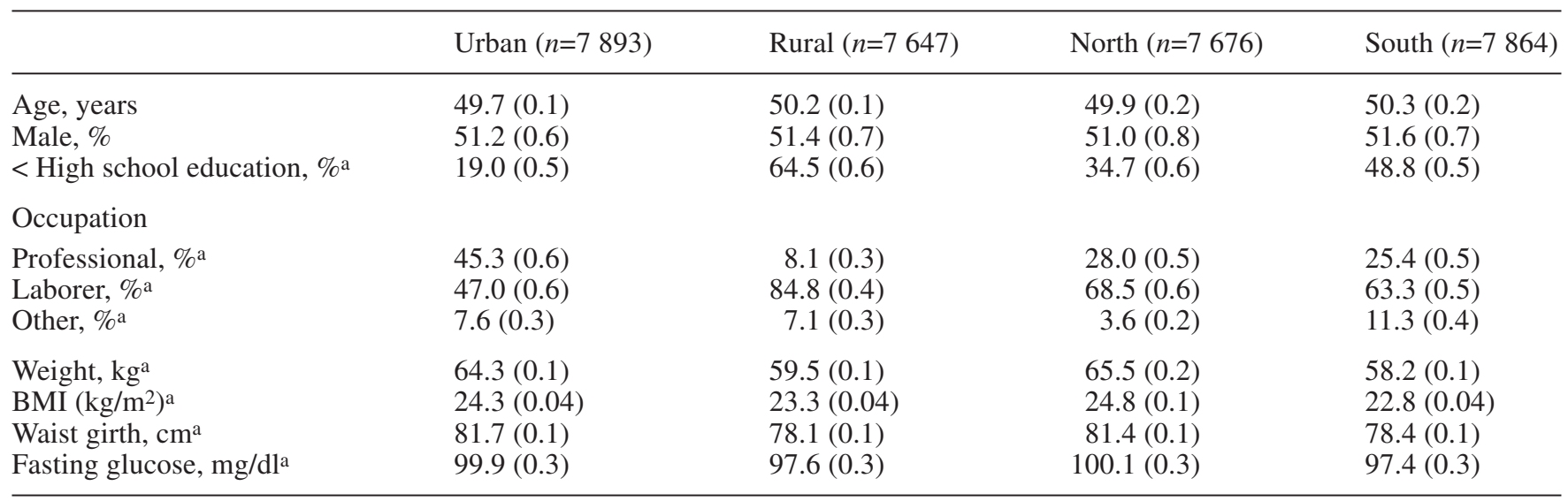

Values are expressed as means or percentages $\pm \mathrm{SE}$

aAge-adjusted to the 2000 China adult population

Census. All data analyses were conducted using STATA 7.0 (Statistics/Data Analysis) software [13]. A $p$ value less than 0.05 was considered statistically significant.

\section{Results}

Table 1 presents demographic and biological characteristics of the study participants for northern and southern China and urban and rural areas. Residents living in urban areas were more educated, more likely to have professional occupations, had a greater BMI and waist girth, and had higher mean fasting serum glucose concentrations than their counterparts living in rural areas of China (all $p<0.001$ ). Residents living in northern China were more likely to have professional occupations, had a greater BMI and waist girth, and had higher mean fasting serum glucose concentrations than their counterparts living in southern China (all $p<0.001$ ).

Prevalence. Prevalence of self-reported diabetes increased with age among men and women, but decreased slightly among women after the age of 65 years (Table 2). The age-standardized prevalence of self-reported diabetes was similar between men and women $(2.1 \%$ vs $1.8 \% ; p=0.24)$. The age-standardized prevalence of self-reported diabetes was higher among men and women living in urban compared to their counterparts in rural areas $(3.3 \%$ vs $0.9 \%$ for men and $2.4 \%$ vs $1.1 \%$ for women; both $p<0.001)$. The agestandardized prevalence of self-reported diabetes was higher among men and women living in northern compared to their counterparts in southern China ( $2.7 \%$ vs $1.4 \%, p=0.001$ for men and $2.3 \%$ vs $1.3 \%$, $p=0.004$ for women).

Prevalence of undiagnosed diabetes increased with age among men and women, although there was a slight decline among men after the age of 65 years (Table 3$)$. The age-standardized prevalence was slightly
Table 2. Prevalence of self-reported history of diabetes in the adult population aged 35-74 years in China, 2000-2001

\begin{tabular}{|c|c|c|c|}
\hline Age, yrs & $\begin{array}{l}\text { Men } \\
\text { Percent } \\
(\mathrm{SE})\end{array}$ & $\begin{array}{l}\text { Women } \\
\text { Percent } \\
\text { (SE) }\end{array}$ & $\begin{array}{l}\text { Total } \\
\text { Percent } \\
(\mathrm{SE})\end{array}$ \\
\hline \multicolumn{4}{|l|}{ Total } \\
\hline $35-44$ & $0.4(0.1)$ & $0.5(0.2)$ & $0.4(0.1)$ \\
\hline $45-54$ & $1.7(0.4)$ & $1.0(0.3)$ & $1.4(0.2)$ \\
\hline $55-64$ & $2.1(0.4)$ & $3.2(0.6)$ & $2.6(0.4)$ \\
\hline $65-74$ & $2.3(0.5)$ & $1.9(0.4)$ & $2.1(0.3)$ \\
\hline $35-74$ & $1.3(0.2)$ & $1.3(0.2)$ & $1.3(0.1)$ \\
\hline Age-Standardized & $2.1(0.2)$ & $1.8(0.2)$ & $1.9(0.1)$ \\
\hline \multicolumn{4}{|l|}{ Urban } \\
\hline $35-44$ & $0.7(0.2)$ & $0.6(0.2)$ & $0.6(0.2)$ \\
\hline $45-54$ & $3.6(0.7)$ & $2.3(0.5)$ & $3.0(0.4)$ \\
\hline $55-64$ & $6.3(1.0)$ & $4.5(0.8)$ & $5.4(0.6)$ \\
\hline $65-74$ & $6.5(1.3)$ & $5.3(1.2)$ & $5.9(0.9)$ \\
\hline $35-74$ & $3.2(0.3)$ & $2.4(0.3)$ & $2.8(0.2)$ \\
\hline Age-Standardized & $3.3(0.3)$ & $2.4(0.3)$ & $2.8(0.2)$ \\
\hline \multicolumn{4}{|l|}{ Rural } \\
\hline $35-44$ & $0.3(0.2)$ & $0.5(0.2)$ & $0.4(0.1)$ \\
\hline $45-54$ & $1.2(0.4)$ & $0.7(0.3)$ & $1.0(0.3)$ \\
\hline $55-64$ & $1.0(0.4)$ & $2.8(0.7)$ & $1.9(0.4)$ \\
\hline $65-74$ & $1.3(0.6)$ & $1.2(0.4)$ & $1.2(0.4)$ \\
\hline $35-74$ & $0.8(0.2)$ & $1.1(0.2)$ & $0.9(0.1)$ \\
\hline Age-Standardized & $0.9(0.2)$ & $1.1(0.2)$ & $1.0(0.1)$ \\
\hline \multicolumn{4}{|l|}{ North } \\
\hline $35-44$ & $0.5(0.2)$ & $0.6(0.2)$ & $0.5(0.2)$ \\
\hline $45-54$ & $2.5(0.6)$ & $1.2(0.3)$ & $1.8(0.3)$ \\
\hline $55-64$ & $3.2(0.7)$ & $4.4(1.1)$ & $3.8(0.7)$ \\
\hline $65-74$ & $3.4(0.8)$ & $3.7(1.0)$ & $3.6(0.6)$ \\
\hline $35-74$ & $1.9(0.3)$ & $1.8(0.3)$ & $1.9(0.2)$ \\
\hline Age-Standardized ${ }^{\mathrm{a}}$ & $2.7(0.3)$ & $2.3(0.3)$ & $2.5(0.2)$ \\
\hline \multicolumn{4}{|l|}{ South } \\
\hline $35-44$ & $0.3(0.2)$ & $0.4(0.2)$ & $0.3(0.1)$ \\
\hline $45-54$ & $1.2(0.4)$ & $0.9(0.4)$ & $1.0(0.3)$ \\
\hline $55-64$ & $1.2(0.4)$ & $2.3(0.7)$ & $1.0(0.3)$ \\
\hline $65-74$ & $1.4(0.7)$ & $0.7(0.2)$ & $1.0(0.4)$ \\
\hline $35-74$ & $0.9(0.2)$ & $0.9(0.2)$ & $0.9(0.1)$ \\
\hline Age-Standardized & $1.4(0.2)$ & $1.3(0.2)$ & $1.4(0.2)$ \\
\hline
\end{tabular}

aAge-adjusted to the 2000 China population 
Table 3. Prevalence of undiagnosed diabetes and impaired fasting glucose ${ }^{\mathrm{a}}$ in the adult population aged 35-74 years in China, 2000-2001

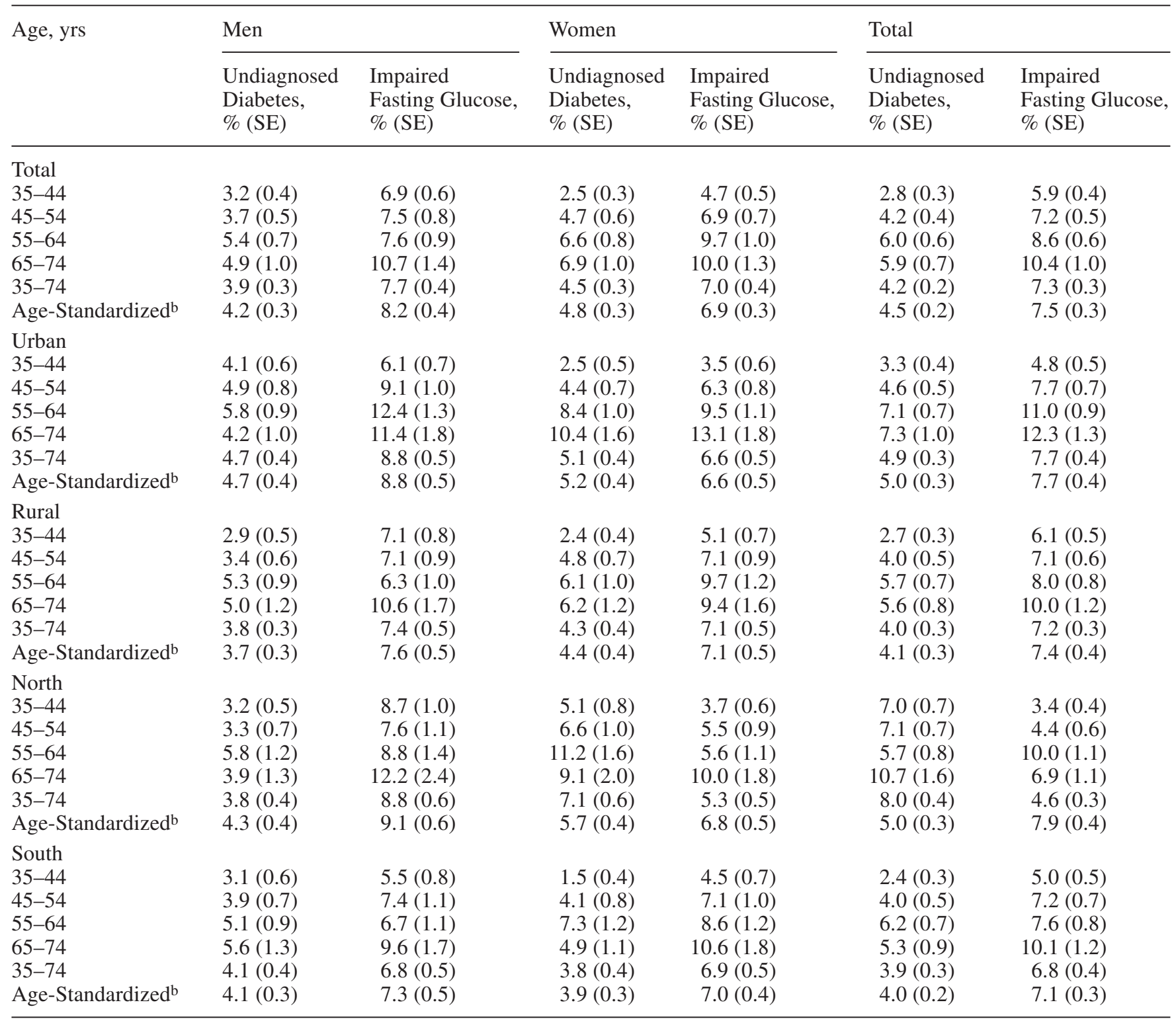

a Based on 2002 American Diabetes Association fasting plasma glucose diagnostic criteria. Undiagnosed diabetes is de-

higher in women than in men, but the difference was not statistically significant $(4.8 \%$ vs $4.2 \%$; $p=0.10)$. The age-standardized prevalence of undiagnosed diabetes was higher in urban than in rural residents $(5.0 \%$ vs $4.1 \% ; p=0.02)$, and in the northern than in the southern residents $(5.0 \%$ vs $4.0 \%$; $p=0.007)$.

The prevalence of impaired fasting glucose increased with age among men and women. The agestandardized prevalence was higher in men than in women $(8.2 \%$ vs $6.9 \% ; p=0.008)$. The age-standardized prevalence of impaired fasting glucose was similar in urban and rural residents $(7.7 \%$ vs $7.4 \%$; $p=0.48)$ and in residents of northern and southern China $(7.9 \%$ vs $7.1 \% ; p=0.10)$. fined as a fasting serum glucose $\geq 7.0 \mathrm{mmol} / \mathrm{l}$; impaired fasting glucose is defined as a fasting serum glucose $6.1-6.9 \mathrm{mmol} / \mathrm{l}$ $\mathrm{b}$ Age-adjusted to the 2000 China population

The age-standardized prevalence of diabetes plus impaired fasting glucose was higher in urban men $(16.7 \%)$ than in urban women $(14.2 \%)$ but similar in rural men (12.2\%) and women (12.6\%) (Fig. 1). The age-standardized prevalence of diabetes was $7.8 \%$ among urban residents and $5.1 \%$ among rural residents $(p<0.001)$. The age-standardized prevalence of diabetes did not differ between men and women in urban $(8.0 \%$ vs $7.6 \% ; p=0.33)$ and rural $(4.6 \%$ vs $5.6 \% ; p=0.08)$ areas. The age-standardized prevalence of diabetes was $7.4 \%$ in northern China compared with $5.4 \%$ in southern China $(p<0.001)$. The age-standardized prevalence was not different between men and women in northern China $(7.0 \%$ vs 


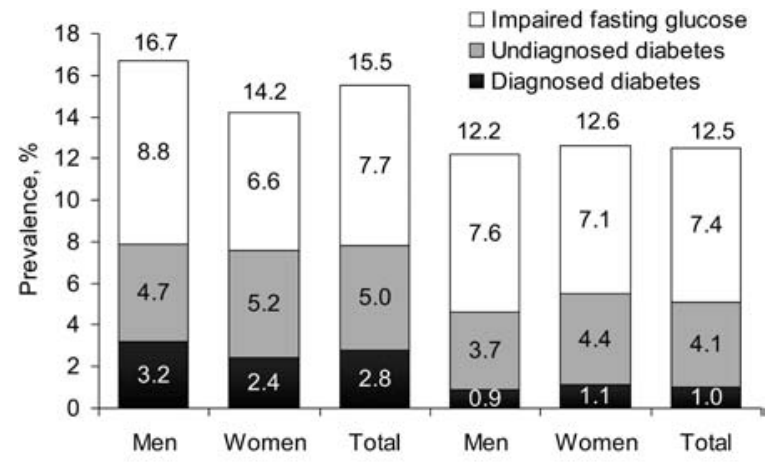

A

Urban

Rural

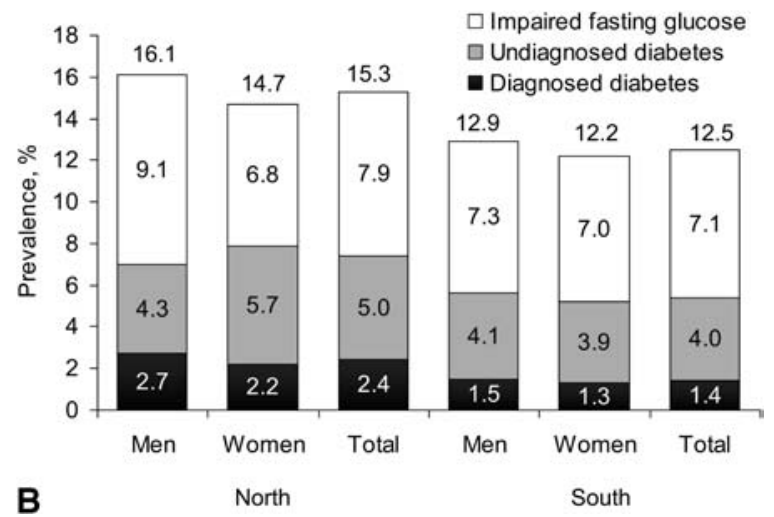

Fig. 1A, B. Age-standardized prevalence of diagnosed and undiagnosed diabetes and impaired fasting glucose among Chinese men and women aged $35-74$ years in northern and southern China and urban and rural areas. Diagnosed diabetes was defined as a self-reported history of diabetes, undiagnosed diabetes by fasting serum glucose $\geq 7.0 \mathrm{mmol} / \mathrm{l}$, and impaired fasting glucose by fasting plasma glucose $6.1-6.9 \mathrm{mmol} / \mathrm{l}$

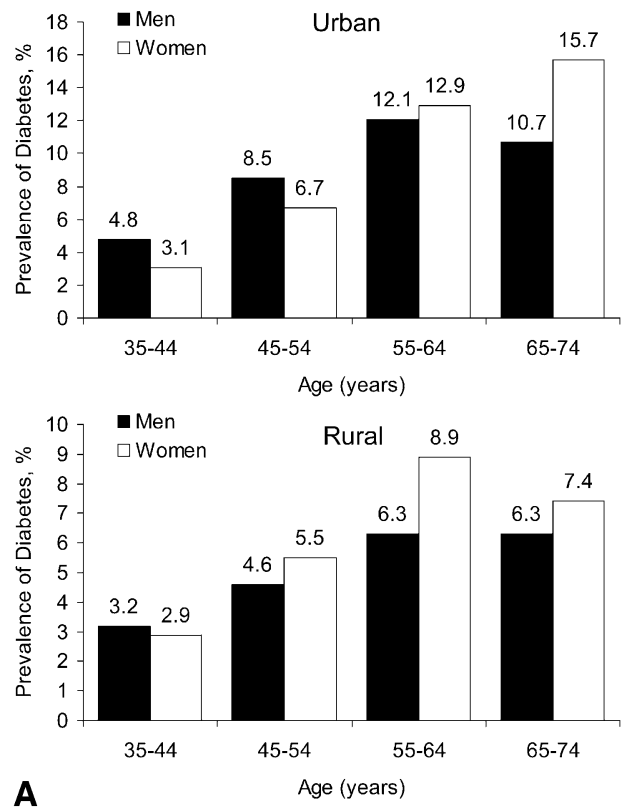

Fig. 2A, B. Prevalence of diabetes among Chinese men and women aged 35-74 years in northern and southern China and urban and rural areas. Diabetes was defined as a self-reported
$7.9 \% ; p=0.16)$ or in southern China $(5.6 \%$ vs $5.2 \%$; $p=0.47$ ).

The prevalence of diabetes (self-reported plus undiagnosed diabetes) increased with age among men and women, although there was a decline among urban men and rural women and among men in the north and women in the south after the age of 65 years and no change among rural men after the age of 65 years (Fig. 2). Women aged 65 to 74 years had the highest prevalence of diabetes $(15.7 \%)$ in urban areas while women aged 55 to 64 years had the highest prevalence $(8.9 \%)$ in rural areas. The prevalence of diabetes was higher for each age group among urban residents compared to rural residents and among northern residents compared to southern residents.

The last national diabetes survey in China was conducted in 1994 [10]. Compared to the findings in that study, our data indicate that the prevalence of diabetes has increased from $1.7 \%$ to $3.2 \%$ (an $88 \%$ increase), $4.1 \%$ to $5.6 \%$ (a $37 \%$ increase), and $7.7 \%$ to $8.6 \%$ (a $12 \%$ increase) in adults aged 35 to 44,45 to 54 , and 55 to 64 , respectively, during the past decade (Fig. 3).

Mean glucose concentrations. Mean glucose concentrations were calculated after excluding participants on hypoglycaemic medication. Overall, women had higher age-standardized fasting serum glucose concentrations than men ( 5.51 vs $5.45 \mathrm{mmol} / \mathrm{l} ; p=0.02$ ). The age-specific mean serum glucose concentrations were 5.33, 5.38, 5.46, and $5.63 \mathrm{mmol} / \mathrm{l}$ in men and 5.25, 5.48, $5.68,5.68 \mathrm{mmol} / \mathrm{l}$ in women aged 35 to 44,45 to 54,55 to 64 , and 65 to 74 years, respectively. The age-standardized mean fasting serum glucose was $5.54 \mathrm{mmol} / \mathrm{l}$

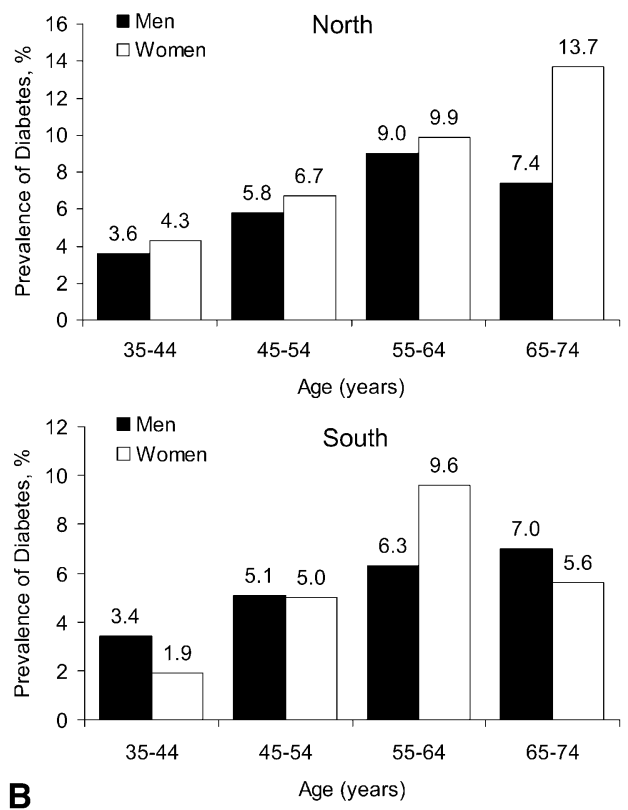

history of diabetes (diagnosed) or fasting plasma glucose $\geq 7.0 \mathrm{mmol} / \mathrm{l}$ (undiagnosed) 


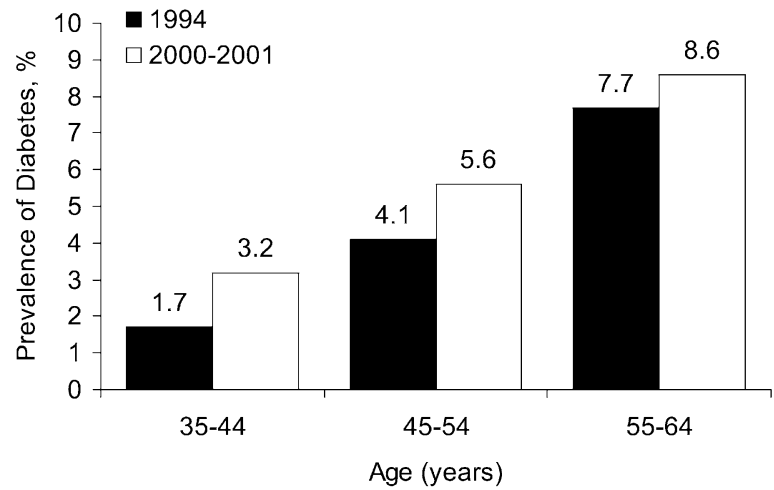

Fig. 3. Prevalence of diabetes among Chinese adults aged 35-64 years in the 1994 Chinese National Survey (10) and 2000-2001 InterASIA Study

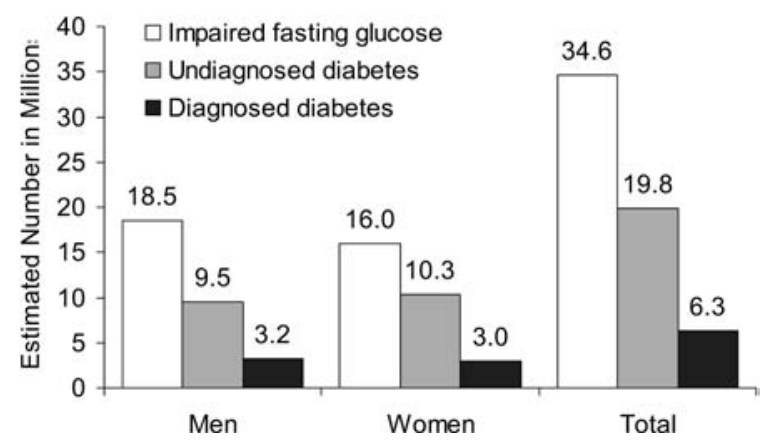

Fig. 4. Age-standardized estimated number with diagnosed and undiagnosed diabetes and impaired fasting glucose among Chinese men and women aged 35-74 years. Diagnosed diabetes was defined as a self-reported history of diabetes, undiagnosed diabetes by fasting serum glucose $\geq 7.0 \mathrm{mmol} / \mathrm{l}$, and impaired fasting glucose by fasting serum glucose $6.1-6.9 \mathrm{mmol} / \mathrm{l}$

among urban residents and $5.42 \mathrm{mmol} / \mathrm{l}$ among rural residents $(p<0.001)$. Age-standardized mean fasting serum glucose values were higher in residents living in northern China than in their counterparts in southern China (5.56 vs $5.41 \mathrm{mmol} / \mathrm{l} ; p<0.001)$.

Estimated number of adults with diabetes and impaired fasting glucose. Based on our prevalence estimates and the number of adults in the Chinese population for the year 2000, the number of adults aged 35 to 74 years in China with diagnosed and undiagnosed diabetes and impaired fasting glucose is estimated to be 6.3 million, 19.8 million, and 34.6 million, respectively (Fig. 4). Of those with diabetes, $75.9 \%$ are undiagnosed. The total number of adults aged 35 to 74 years with diabetes and impaired fasting glucose is estimated to be 60.5 million (31.2 million men and 29.3 million women).

\section{Discussion}

Overall, the InterASIA results indicate that $26.1 \mathrm{mil}-$ lion (or 5.5\%) adults aged 35 to 74 years in China have diabetes. Of them, approximately 19.8 million (or $76 \%$ ) are undiagnosed. In addition, 34.6 million (or $7.3 \%$ ) adults aged 35 to 74 years were found to have an impaired fasting glucose, which is an important risk factor in the development of clinical diabetes.

InterASIA is the first national study to be conducted in China using current diabetes diagnostic criteria by the ADA. This study provides the most reliable and up-to-date information on the prevalence of diabetes in the adult population of China. This study was conducted in a large representative sample of the Chinese adult population in which a high response rate was achieved. Standard protocols and instruments along with strict training processes for data collection and a vigorous quality assurance programmme were used to ensure the quality of the data we collected. In addition, standard laboratory methods for the measurement of glucose were used and a central clinical laboratory carried out all of the glucose assays. For all these reasons, the InterASIA data probably provides the most accurate and precise estimates of diabetes to date in the general Chinese adult population.

Our findings have important public health implications. Diabetes increases the risk of micro- and macrovascular complications in the general population and results in an enormous economic burden to society [1, $2,7,14,15]$. In addition, impaired fasting glucose and impaired glucose tolerance have been identified as risk factors for overt diabetes and cardiovascular disease [16, 17, 18]. Randomized clinical trials have shown that diet and exercise interventions reduce the incidence of diabetes in people with impaired glucose tolerance $[19,20]$. The present study indicates that a large number of Chinese adults have diabetes or impaired fasting glucose and portends the urgent need to establish a national education programmme in China to promote regular community and clinic-based diabetes screening for the early detection of diabetes and impaired fasting glucose (or impaired glucose tolerance) and to prevent and treat diabetes for the reduction of diabetes-related complications in the general population.

For epidemiological study purposes, the ADA recommends the use of a fasting glucose concentration of $7.0 \mathrm{mmol} / \mathrm{l}$ solely for a diagnosis of diabetes whereas, the new criteria adopted by WHO recommends the same fasting glucose concentration $(7.0 \mathrm{mmol} / \mathrm{l})$ but also incorporates the use of the 2-h glucose tolerance test with a concentration of at least $11.1 \mathrm{mmol} / \mathrm{l}$ for a diabetes diagnosis $[11,21]$. For both, a clinical diagnosis of diabetes for an individual cannot be established from a single blood glucose measurement. The diagnosis of diabetes in an individual must be confirmed on a subsequent day.

One limitation of our study is that a 2 -h glucose tolerance test was not conducted. Therefore, we are not able to use WHO criteria for the diagnosis of diabetes. In addition, several epidemiological studies in- 
dicate that impaired fasting glucose might not be as sensitive as impaired glucose tolerance as a predictor of diabetes $[22,23]$. Furthermore, the ADA and WHO criteria might identify different groups of individuals [24, 25]. In an analysis of 16 different populations in the Diabetes Epidemiology: Collaborative Analysis of Diagnostic Criteria in Europe (DECODE), the WHO criteria were more likely to diagnose diabetes in lean individuals, while the ADA criteria were more likely to identify those in middle-aged, obese individuals [24]. Moreover, only $46 \%$ of those with diabetes according to the ADA criteria actually met the WHO 2-h glucose criterion [24]. The OGTT has the potential to identify individuals who have abnormal glucose concentrations and increased cardiovascular risk who would not be detected by fasting glucose alone. However, the OGTT has been found to have poor reproducibility [26, 27]. Intra-individual coefficients of variation were $6.5 \%$ for the fasting plasma glucose and $16.7 \%$ for the 2 -h plasma glucose after OGTT in the Hoorn Study [26].

Estimates of diabetes prevalence have been reported in many population-based studies $[3,7]$. Due to the extent to which selection of the sample, measurement methods, and choice of diagnostic criteria and definitions of diabetes influence prevalence estimation, caution should be observed in comparisons of the findings shown here to corresponding data from previous surveys. The dramatic increase of prevalence from the 1994 survey to our study among the younger age group could be the result of changes in lifestyle factors among this group. In addition, the higher ratio of undiagnosed to diagnosed cases is likely due to both a lack of population-based screening programmmes and a relatively rapid and recent increase in the incidence of diabetes. These data portend the need for screening and prevention programmmes that begin at a young age.

An OGTT was used to determine the prevalence of diabetes in the 1994 survey according to the 1985 WHO criteria. In our study, fasting serum glucose was used to diagnose new cases of diabetes. Our approach is likely to have fallen short of an estimate of diabetes prevalence based upon both a fasting plasma glucose and the 2-h OGTT test value [11, 28, 29]. Based on data from NHANES III, the estimated prevalence of diabetes among participants without a medical history of diabetes in the U.S. was $6.34 \%$ using 1985 WHO criteria and $4.35 \%$ using ADA criteria [11]. Data collected in the Hong Kong Cardiovascular Risk Factor Prevalence study indicates that the prevalence of diabetes (self-reported plus undiagnosed) was 6.2\%, 9.2\%, and $9.8 \%$ based on ADA and WHO 1985 and WHO 1998 criteria, respectively [28]. Fasting glucose is recommended over the OGTT in epidemiological studies and for individual diagnostic purposes for its ease, convenience, and acceptability among participants and for its reproducibility and cost-effectiveness [11]. More likely, the true increase in the prevalence of diabetes in China could be even greater than our reported results.

The prevalence of diabetes in China estimated in this study is lower than that noted in other developing countries in Asia [7, 28, 30, 31]. The 1996 diabetes survey conducted in southern Taiwan identified a crude prevalence of diabetes, by the ADA criteria, in adults aged 20 years and older of $9.0 \%$ [30]. The prevalence of diabetes among Singaporean residents aged 18 to 69 years was estimated to be $8.1 \%$ in 1998 , using WHO criteria [31]. In Hong Kong, the Cardiovascular Risk Factor Prevalence Study conducted from 1995 to 1996 yielded a prevalence of diabetes, by WHO criteria, of $9.8 \%$ in adults aged 25 to 74 years [28].

A higher prevalence of diabetes among urban residents compared to rural residents has been observed throughout the world [7, 10, 32]. The prevalence of diabetes among urban residents in China estimated in our study was higher than in rural residents although the prevalences of impaired fasting glucose were similar. Urbanization is associated with changes in a number of lifestyle factors, such as physical inactivity, unhealthy diet, and obesity, which are implicated in the aetiology of diabetes. Epidemiological studies conducted in China have documented a higher mean BMI and prevalence of obesity in urban compared to rural areas $[33,34]$. Our study showed that the entire distribution of serum glucose was shifted to higher concentrations among urban residents. Therefore, a much larger urban-rural difference in the relative prevalence of diabetes is observed while a smaller difference in relative prevalence but a larger difference in the absolute number of impaired fasting glucose cases is seen.

In summary, our results indicate that diabetes is very common in the Chinese adult population and is probably rising in both incidence and prevalence. The prevalence of diabetes is higher in northern than in southern China and in urban compared with rural areas. Three out of every four people with diabetes are undiagnosed, indicative of a lack of population-based diabetes screening programmmes and a relatively rapid and recent increase in incidence of diabetes. These results underscore the urgency for development of national strategies, which must cover both urban and rural communities, to improve detection, prevention and treatment of diabetes in China in order to avert an epidemic of diabetes and its complications.

\section{Members of the InterASIA Collaborative Group}

Steering Committee Members. J. He (Co-Principal Investigator), P.K. Whelton (Co-Principal Investigator), D. Glasser, D. Gu, S. MacMahon, B. Neal, R. Patni, R. Reynolds, P. Suriyawongpaisal, X. Wu, X. Xin, and X.-H. Zhang. 
Participating Institutes and Principal Staff. Tulane University, New Orleans, LA., USA-Drs. J. He (PI), L.A. Bazzano, J. Chen, P. Muntner, K. Reynolds, P.K. Whelton, and X. Xin; University of Sydney, Sydney, Australia-Drs. S. MacMahon (PI), N. Chapman, B. Neal, M. Woodward and X.-H. Zhang.

China:

Fuwai Hospital and Cardiovascular Institute, Chinese Academy of Medical Sciences and Peking Union Medical College-Drs. D. Gu (PI), X. Wu, W. Gan, S. Su, D. Liu, X. Duan, G. Huang.

Beijing-Y. Ma, X. Liu, Z. Tian, X. Wang, G. Fan, J. Wang, C. Qiu.

Fujian-L. Yu, X. Pu, X. Bai, L. Li, W. Wu.

Jilin-L. Xu, J. Liu, Y. Jiang, Y. Lan, L. Huang, H. Yin.

Sichuan-X. Wu, Y. Deng, J. He, N. Zhang, X. Yang.

Shandong-X. Chen, R. Wei, X. Liu, H. Ruan, M. Li, C. Zhang.

Guangxi-N. Chen, X. Meng, F. Wei, Y. Xu.

Qinghai-T. Wu, J. Ji, C. Shi, P. Yang.

Hubei-L. Wang, Y. Hu, L. Yan, Y. Wang.

Jiangsu-C. Yao, L. Ma, J. Zhang, M. Xu, Z. Zhou.

Shanxi-J. Mu, Z. Wang, H. Li, Z. Zhao.

Thailand:

Mahidol University-Drs. P. Suriyawongpaisal (PI),

S. Cheepudomwit, P. Loelekla, P. Srithara; Bangkok: Drs. P. Sariyaporn, P. Pongchoke, S. Jaiyavat, C. Nantawan, V. Kasikoson; North: Drs. S. Thamthitiwat, P. Siviroj, J. Suwanteerangkul; Northeast: Drs. P. Tasanavivat, P. Kessomboon, Ms. S. Horas; South: Drs. V. Chongsuvivatwong, T. Yipintsoi, N. Apakupakul, W. Jirathamopas, W. Jintapakorn; Central: Drs. V. Kosulwat, A. Boonpraderm, A. Wongchanapai, C. Wanijjakul.

Acknowledgements. The InterASIA study was funded by a contractual agreement between Tulane University, L.A., USA and Pfizer Inc., N.Y., USA. Several researchers employed by Pfizer Inc. were members of the Study Steering Committee that designed the study. However, the study was conducted, analyzed, and interpreted by the investigators independently of the sponsor.

\section{References}

1. Haffner SM, Lehto S, Ronnemaa T, Pyorala K, Laakso M (1998) Mortality from coronary heart disease in subjects with type 2 diabetes and in nondiabetic subjects with and without prior myocardial infarction. $\mathrm{N}$ Engl $\mathrm{J}$ Med 339:229-234

2. Brancati FL, Whelton PK, Randall BL, Neaton JD, Stamler J, Klag MJ (1997) Risk of end-stage renal disease in diabetes mellitus: a prospective cohort study of men screened for MRFIT. Multiple Risk Factor Intervention Trial. JAMA 278:2069-2074

3. Harris MI, Flegal KM, Cowie CC et al. (1998) Prevalence of diabetes, impaired fasting glucose, and impaired glucose tolerance in U.S. adults. The Third National Health and
Nutrition Examination Survey, 1988-1994. Diabetes Care 21:518-524

4. Burt VL, Cutler JA, Higgins M et al. (1995) Trends in the prevalence, awareness, treatment, and control of hypertension in the adult US population. Data from the health examination surveys, 1960 to 1991. Hypertension 26:60-69

5. Sempos C, Fulwood R, Haines C et al. (1989) The prevalence of high blood cholesterol levels among adults in the United States. JAMA 262:45-52

6. Mokdad AH, Bowman BA, Ford ES, Vinicor F, Marks JS, Koplan JP (2001) The continuing epidemics of obesity and diabetes in the United States. JAMA 286:1195-200

7. King H, Aubert RE, Herman WH (1998) Global burden of diabetes, 1995-2025: prevalence, numerical estimates, and projections. Diabetes Care 21:1414-1431

8. Li G, Hu Y, Pan X (1996) Prevalence and incidence of NIDDM in Daqing City. Chin Med J 109:599-602

9. Hu YH, Li GW, Pan XR (1993) Incidence of NIDDM in Daqing and forecasting of NIDDM in China in 21st century. Chin J Int Med 32:173-175

10. Pan XR, Yang WY, Li GW, Liu J (1997) Prevalence of diabetes and its risk factors in China, 1994. Diabetes Care 20:1664-1669

11. Expert Committee on the Diagnosis and Classification of Diabetes Mellitus (2002) Report of the Expert Committee on the Diagnosis and Classification of Diabetes Mellitus. Diabetes Care 25 [Suppl 1]:S5-20

12. National Center for Health Statistics (1994) Plan and operation of the Third National Health and Nutrition Examination Survey, 1988-1994. Vital Health Stat 1 32:1-407

13. StataCorp (2001) Stata Statistical Software: Release 7.0. College Station. Stata, TX

14. National Institutes of Health, National Institute of Diabetes and Digestive and Kidney Diseases (1995) Diabetes in America, 2nd edn. U.S. Govt. Printing Office, Washington, DC (NIH publ. no. 95-1468)

15. Chi ZS, Lee ET, Lu M, Keen H, Bennett PH and the WHO Multinational Study Group (2001) Vascular disease prevalence in diabetic patients in China: standardized comparison with the 14 centres in the WHO multinational study of vascular disease in diabetes. Diabetologia 44 [Suppl 2]:S82-S86

16. Fuller JH, Shipley MJ, Rose G, Jarrett RJ, Keen H (1980) Coronary-heart-disease risk and impaired glucose tolerance. The Whitehall study. Lancet 1:1373-1376

17. Vegt F de, Dekker JM, Jager A et al. (2001) Relation of impaired fasting and postload glucose with incident type 2 diabetes in a Dutch population. The Hoorn Study. JAMA 285:2109-2113

18. Pan XR, Hu YH, Li GW, Liu PA, Bennett PH, Howard BV (1993) Impaired glucose tolerance and its relationship to ECG-indicated coronary heart disease and risk factors among Chinese: Da Qing IGT and diabetes study. Diabetes Care 16:150-156

19. Pan XR, Li GW, Hu YH et al. (1997) Effects of diet and exercise in preventing NIDDM in people with impaired glucose tolerance: the Da Qing IGT and Diabetes Study. Diabetes Care 20:537-544

20. Knowler WC, Barrett-Connor E, Fowler SE et al. (2002) Reduction in the incidence of type 2 diabetes with lifestyle intervention or metformin. N Engl J Med 346:393-403

21. Alberti KGMM, Zimmet PZ for the WHO Consultation (1998) Definition, diagnosis, and classification of diabetes mellitus and its complications. Part 1: diagnosis and classification of diabetes mellitus. Provisional report of a WHO Consultation. Diabet Med 15:539-553 
22. Shaw JE, Zimmet PZ, de Courten M et al. (2000) Impaired fasting glucose or impaired glucose tolerance. What best predicts future diabetes in Mauritius? Diabetes Care 23:714-715

23. Gabir MM, Hanson RL, Dabelea D et al. (2000) The 1997 American Diabetes Association and 1999 World Health Organization criteria for hyperglycemia in the diagnosis and prediction of diabetes. Diabetes Care 23:1108-1112

24. Decode Study Group (1998) Will new diagnostic criteria for diabetes mellitus change phenotype of patients with diabetes? Reanalysis of European epidemiological data. BMJ 317:371-375

25. Wahl PW, Savage PJ, Psaty BM, Orchard TJ, Robbins JA, Tracy RP (1998) Diabetes in older adults: comparison of 1997 American Diabetes Association classification of diabetes mellitus with 1985 WHO classification. Lancet 352:1012-1015

26. Mooy JM, Gootenhuis PA, de Vries H et al. (1996) Intraindividual variation of glucose, specific insulin and proinsulin concentrations measured by two oral glucose tolerance tests in general Caucasian population: the Hoorn Study. Diabetologia 39:298-305

27. Ko GT, Chan JC, Woo J, Cockram CS (1998) Use of the 1997 American Diabetes Association diagnostic criteria for diabetes in a Hong Kong Chinese population. Diabetes Care 21:2094-2097
28. Janus ED, Watt NM, Lam KS et al. (2000) The prevalence of diabetes, association with cardiovascular risk factors and implications of diagnostic criteria (ADA 1997 and WHO 1998) in a 1996 community-based population study in Hong Kong Chinese. Diabet Med 17:741-745

29. Choi KM, Lee J, Kim DR et al. (2002) Comparison of ADA and WHO criteria for the diagnosis of diabetes in elderly Koreans. Diabet Med 19:853-857

30. Lu FH, Yang YC, Wu JS, Wu CH, Chang CJ (1998) A population-based study of the prevalence and associated factors of diabetes mellitus in southern Taiwan. Diabet Med 15:564-572

31. Cutter J, Tan BY, Chew SK (2001) Levels of cardiovascular disease risk factors in Singapore following a national intervention programme. Bull World Health Organ 79:908-915

32. Singh RB, Bajaj S, Niaz MA, Rastogi SS, Moshiri M (1998) Prevalence of type 2 diabetes mellitus and risk of hypertension and coronary artery disease in rural and urban population with low rates of obesity. Int J Cardio 66:65-72

33. Wu Y, Zhou B, Tao S et al. (2002) Prevalence of overweight and obesity in Chinese middle-aged populations: current status and trend of development. Chinese J Epidem 23:11-15

34. Zhai S, McGarvey ST (1992) Temporal changes and ruralurban differences in cardiovascular disease risk factors and mortality in China. Hum Biol 64:807-819 